\title{
What validation tests can be done by the clinical medical physicist while waiting for the standardization of quantitative SPECT/CT imaging?
}

Hanna Piwowarska-Bilska ( $\sim$ hanna.piwowarska.bilska@gmail.com )

Pomeranian Medical University in Szczecinie https://orcid.org/0000-0003-3617-7687

\section{Aleksandra Supińska}

Voivodship Hospital in Gorzow Wielkopolski: Wielospecjalistyczny Szpital Wojewodzki w Gorzowie Wielkopolskim

\section{Bożena Birkenfeld}

Pomeranian Medical University in Szczecin: Pomorski Uniwersytet Medyczny w Szczecinie

\section{Original research}

Keywords: absolute quantification, SPECT/CT, absolute SUV

Posted Date: December 2nd, 2020

DOl: https://doi.org/10.21203/rs.3.rs-118913/v1

License: (c) (i) This work is licensed under a Creative Commons Attribution 4.0 International License. Read Full License

Version of Record: A version of this preprint was published at EJNMMI Physics on February 5th, 2022. See the published version at https://doi.org/10.1186/s40658-022-00434-6. 


\section{Title}

What validation tests can be done by the clinical medical physicist while waiting for the standardization of quantitative SPECT/CT imaging?

\section{Authors}

Hanna Piwowarska-Bilska ${ }^{1 *}$, Aleksandra Supińska ${ }^{2}$, Bożena Birkenfeld ${ }^{1}$

1. Nuclear Medicine Department of Pomeranian Medical University in Szczecin, Poland

2. Clinical Nuclear Medicine Department of the University of Zielona Gora, MultiSpecialist Regional Hospital in Gorzow Wielkopolski, Poland

*corresponding author: Hanna Piwowarska-Bilska, hanna.piwowarska.bilska@gmail.com

\section{Abstract \\ Objective}

The aim of the study was to assess the accuracy of quantitative SPECT/CT imaging in a clinical setting and to compare test results from two nuclear medicine departments.

Methods

Phantom studies were carried out with two gamma cameras manufactured by GE Healthcare: Discovery NM/CT 670 and NM/CT 850, used in two nuclear medicine departments.

Results

The convergence of activity concentration recovery was validated for the two gamma cameras operating in two medical centres using a homogeneous 3D phantom. The comparison of results revealed a $5 \%$ difference in the calibration factor $\mathrm{Bg}$. cal; $6 \%$ difference in $\mathrm{COV}$, and a $0.6 \%$ difference in total activity deviation $\Delta$ Atot.

Recovery coefficients (RCmax) for activity concentration in spheres of the anthropomorphic phantom was measured for different image reconstruction techniques. $\mathrm{RC}_{\max }$ was in the range of 0.2-0.4 for the smallest sphere $(\phi 10 \mathrm{~mm})$, and 1.3-1.4 for the largest sphere $(\phi 37 \mathrm{~mm})$.

Conversion factors for SUVmax and SUVmean for the gamma camera systems used were 0.99 and 1.13 , respectively.

\section{Conclusions}


1) Measurements taken in our study confirmed the clinical suitability of 5 parameters of image quality (Bg. cal- background calibration factor, $\triangle$ Atot- total activity deviation, COV- noise

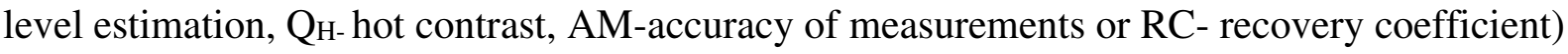
for the validation of SPECT/CT system performance in terms of correct quantitative acquisitions of images. 2) This work shows that absolute SPECT/CT quantification is achievable in clinical nuclear medicine centers. Results variation of quantitative analyzes between centers is mainly related to the use of different reconstruction methods. 3) It is necessary to standardize the technique of measuring the SUV conversion factor obtained with different SPECT/CT scanners.

Keywords: absolute quantification, SPECT/CT, absolute SUV

\section{Background}

The integrated diagnostic SPECT/CT systems with iterative algorithms for image reconstruction allow for the clinical use of quantitative SPECT [1]. So far, a standard methodology for evaluating the performance of quantitative SPECT/CT systems has not been established. SPECT is a quantitative imaging technique and therefore requires a common quality control procedure to maintain the accuracy and precision of quantitation. Repeatability and reproducibility are essential requirements for any quantitative measurement. Repeatability in SPECT relates to the uncertainty in obtaining the same result in the same patient examined more than once on the same system. Reproducibility relates to the variation that results when different conditions are used to make the measurements, for example different gamma camera systems.

Standardized uptake value (SUV) is a widely available and easy-to-use quantifier of radioactivity concentration in SPECT images. Physical and clinical conditions for its measurement still need to be standardized. A measured SUV cannot be used as an absolute number. SUVmax, which is preferred by physicians, depends, among other things, on the chosen SPECT/CT image reconstruction technique.

Selected issues related to the quantitative analysis of SPECT/CT images were addressed in the present study: 1) What tests can be done to assess the performance of a SPECT/CT gamma camera used for quantitative imaging? 2) Is the level of accuracy of activity measurement obtained by manufacturers in their research laboratories achievable in the clinical facilities of nuclear medicine? 3) How can the repeatability of SUV measurements for two different SPECT/CT systems be tested? 


\section{Methods}

The present study was conducted in two departments of nuclear medicine: the Clinical Nuclear Medicine Department of the University of Zielona Gora, Multi-Specialist Regional Hospital in Gorzow Wielkopolski (Department 1), and in the Nuclear Medicine Department of the Pomeranian Medical University in Szczecin (Department 2). Phantom tests were performed using a Discovery NM/CT 670 gamma camera operating in Department 1 and a NM/CT 850 gamma camera operating in Department 2. Acquired data were processed with Xeleris 2 from GE Healthcare.

1. Accuracy of activity recovery in a homogeneous phantom.

A cylindrical homogeneous WCC phantom, $18 \mathrm{~cm}$ high, $20 \mathrm{~cm}$ in diameter and $5640 \mathrm{ml}$ volume, was filled with a homogeneous solution of the radioisotope $(99 \mathrm{mTc})$ with activity of $294 \mathrm{MBq}$ (Department 1) and $295 \mathrm{MBq}$ (Department 2) at the moment of scan start. The same SPECT/CT acquisitions of the phantom were performed in the two medical departments. The acquisition parameters were as follows: low-energy high-resolution collimator; the photopeak emission energy window: 127-155 keV; the scatter window: 108-129 keV. SPECT images were acquired with 60 projections, 20 s/projection. Acquired images were reconstructed using 24 iterations, 4 subsets, Gaussian smoothing filter, and corrections (AC, SC, RR). The following parameters were established for the cylindrical homogeneous phantom, as proposed by Gnesin et al. [2]:

A) Calibration factor (Bg.cal), which is the ratio of the activity concentration measured in the reconstructed SPECT phantom background $\left(a_{c, \mathrm{bg}}\right)$ to the expected activity concentration of liquid filling the phantom $\left(A_{c, \mathrm{bg}}\right)$, measured during phantom preparation.

$$
B g . c a l=\frac{a_{c, b g}}{A_{c, b g}}
$$

$a_{c, \mathrm{bg}}$ was evaluated as the mean value for 5 circular regions of interest of $16 \mathrm{~cm}$ in diameter centred on the cylinder axis of the phantom placed at different axial locations (Figure 1). 


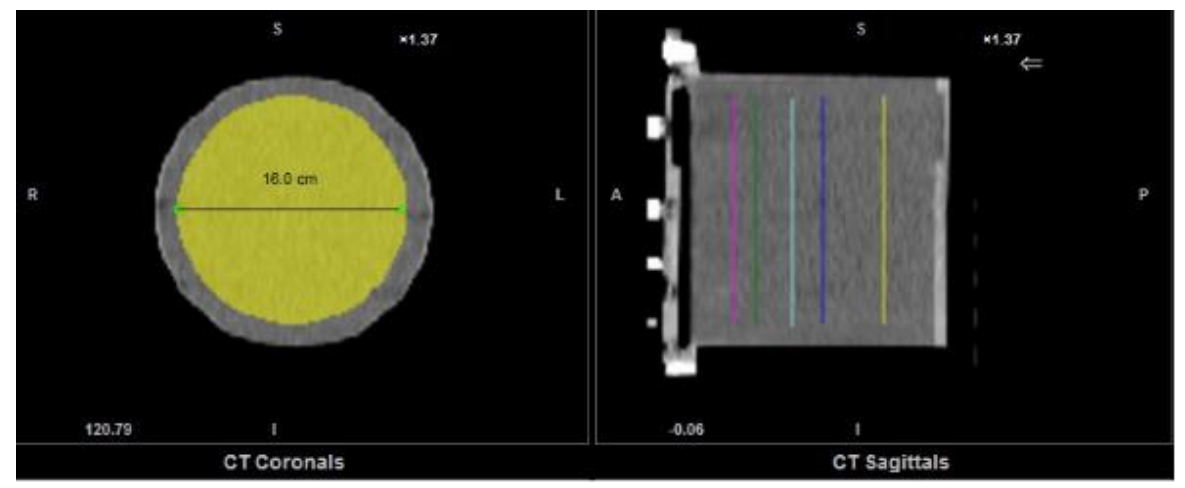

Fig. 1. Image of the WCC phantom with 5 circular regions of interest of $16 \mathrm{~cm}$ in diameter located at different levels along the long axis of the phantom, reconstructed with Xeleris 2 from GE Healthcare.

B) The image noise was evaluated using the coefficient of variation (COV), which is the ratio of standard deviation $\left(\sigma_{\mathrm{bg}}\right)$ to the average signal measured in the phantom background $a_{c, \text { bg. }}$

$$
\operatorname{COV}(\%)=\frac{\sigma_{b g}}{a_{c, b g}} \times 100
$$

C) Total activity deviation $\left(\Delta A_{t o t}(\%)\right)$ was calculated:

$$
\Delta A_{t o t}(\%)=\frac{A_{t o t, r e c}-A_{t o t}}{A_{t o t}} \times 100
$$

where $A$ tot, rec is the total activity in the phantom measured in the image with computer software, and $A_{\text {tot }}$ is the total activity of radioactive liquid in the phantom measured with a meter during phantom preparation.

2. Accuracy of measurements for the activity in hot spherical inserts.

In Department 1 and then in Department 2 identical SPECT/CT acquisitions were performed for the NEMA IEC Body Phantom prepared consistently with instructions on laboratory measurements provided in the white paper published by GE Healthcare [4]. The following acquisition parameters were used: low-energy high-resolution collimator; the photopeak emission energy window: 127-155 keV; the scatter window: 108-129 keV. SPECT acquisitions were acquired with 120 projections, 30 s/projection. The NEMA IEC Body Phantom, loaded 
with a Tc99m solution, was used to assess quantification accuracy using 6 different sphere sizes. Six spherical inserts $(10,13,17,22,28$, and $37 \mathrm{~mm}$ in diameter) were filled with an activity concentration 8 times higher than the activity concentration present in the phantom cylinder.

A) Acquired images were reconstructed using 6 subsequent iterative techniques:

5 iterations, 15 subsets; AC, SC, RR corrections; no filtering,

24 iterations, 8 subsets; AC, SC, RR corrections; no filtering,

4 iterations, 10 subsets; AC, SC, RR corrections; no filtering,

2 iterations, 10 subsets; AC, SC, RR corrections; no filtering,

2 iterations, 10 subsets; AC, SC, RR corrections; Butterworth filter (0.48 10),

4 iterations, 10 subsets; AC correction; no filtering.

Accuracy of activity recovery for 6 spheres in the images of the phantom was evaluated from the formula:

$$
\text { Accuracy }=\left(1-\frac{\mid \text { Loaded activity-Measured activity } \mid}{\text { Loaded activity }}\right) \times 100 \%
$$

where: Loaded activity - real activity measured with an activity meter at the time of acquisition, Measured activity - activity measured in SPECT/CT images with Q.Metrix from GE Healthcare for individual spherical inserts. Figure 2 presents the technique of radioactivity measurement for 6 hot spheres of the phantom during the analysis of reconstructed images.

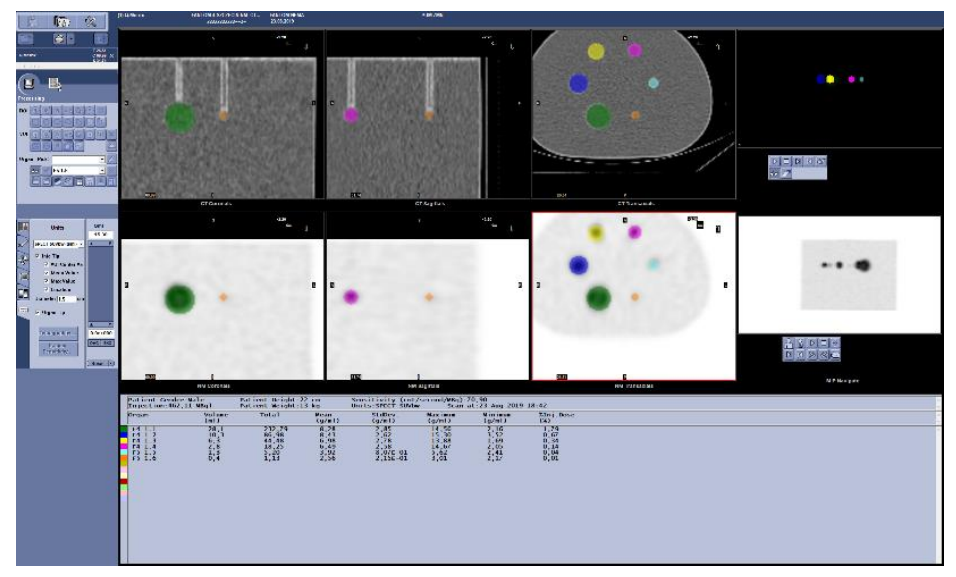

Fig. 2. A screenshot of the Q.Metrix software during the measurement of hot spheres activity in the cross-sections of the anthropomorphic NEMA IEC Body Phantom. Colour rings correspond with VOI (Volume of Interest) for 6 hot spheres.

B) Cross-sections of the anthropomorphic NEMA IEC Body Phantom were additionally reconstructed using 7 subsequent techniques, with 4, 8, 12, 16, 20, 24, 48 iterations; 4 subsets; 
AC, SC, RR corrections; and filtering (Gaussian smoothing of FWHM $7.5 \mathrm{~mm}$ ). Recovery coefficient (RC) was evaluated for all 6 spheres and images acquired with 7 different techniques [2]:

$$
R C_{j, \max }=\frac{a_{c, s p h, j, \max }}{A_{c, s p h}}
$$

where $\mathrm{j}$ - is the number of sphere, $a_{c, s p h, j, \max }$ is maximum activity concentration in the sphere measured with Q.Metrix, $A_{c, s p h}$ is activity concentration in the sphere established based on measurements taken with an activity meter.

The following parameters were also evaluated for the images of the NEMA IEC Body Phantom:

- activity concentration in the background,

- activity concentration in the $22 \mathrm{~mm}$ diameter hot sphere,

- hot contrast $\left(\mathrm{Q}_{\mathrm{H}} 22\right)$ for the $22 \mathrm{~mm}$ diameter sphere, from the formula [2]:

$$
Q_{H, j}(\%)=\frac{\left(a_{c, s p h, j} / a_{c, b g}\right)-1}{\left(A_{c, s p h} / A_{c, b g}\right)-1} \times 100
$$

where $a_{c, b g}$ is activity concentration in the background measured in the image of the phantom, and $A_{c, b g}$ is activity concentration of the radioactive liquid in which hot spheres are immersed (background) measured with an activity meter.

3. Comparison of SUVs acquired for a single calibration source

To determine the conversion factor for SUVmax and SUVmean registered in studies with different gamma cameras, two identical SPECT/CT acquisitions and reconstructions of the same Tc99m calibration source were performed in two nuclear medicine departments. The calibration source was a $22 \mathrm{~mm}$ diameter hot spherical insert of the NEMA/IEC NU2 phantom with activity of $20 \mathrm{MBq}$ at the time of the first scan start.

The acquisition of the calibration source was carried out with the Discovery NM/CT 670 gamma camera in Department 1, and 3 hours later with the NM/CT 850 gamma camera in Department 2 (Figure 3). 


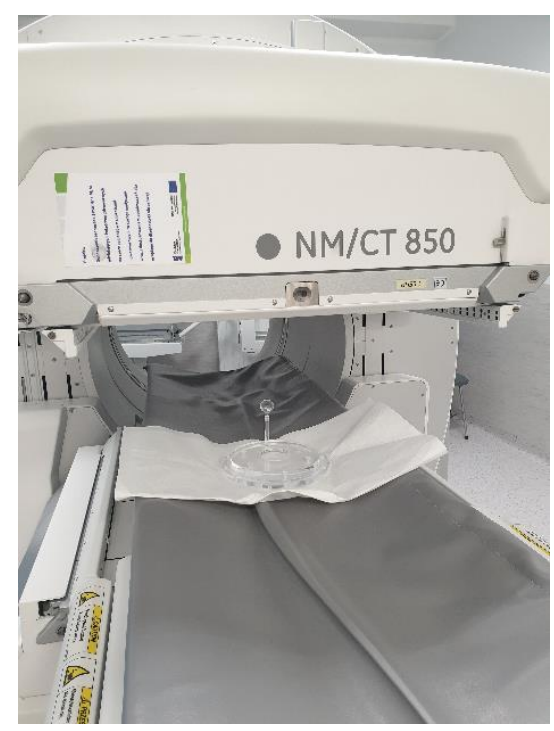

Fig. 3. Acquisition of the calibration source in Department 2.

\section{Results}

1. Accuracy of activity recovery in a cylindrical homogeneous phantom.

Table 1 presents data on the accuracy of activity concentration recovery in the images of a cylindrical homogeneous phantom (WCC phantom) acquired with two gamma cameras in two nuclear medicine departments. The comparison of results for the two gamma cameras revealed a $5 \%$ difference in the calibration factor $\mathrm{Bg}$. cal; $6 \%$ difference in $\mathrm{COV}$, and a $0.6 \%$ difference in total activity deviation $\Delta$ Atot. Activity concentration measured for the $99 \mathrm{mTc}$ solution with an activity meter and then measured in the image with Q.Metrix was identical for both gamma cameras.

\begin{tabular}{|l|c|c|c|c|c|}
\hline Coefficient & $\begin{array}{c}\text { Calibration } \\
\text { factor } \\
\text { Bg. cal }\end{array}$ & $\begin{array}{c}\text { Coefficient of } \\
\text { variation COV } \\
(\%)\end{array}$ & $\begin{array}{c}\text { Total } \\
\text { activity } \\
\text { deviation } \\
\Delta \text { Atot } \\
(\%)\end{array}$ & $\begin{array}{c}\text { Activity } \\
\text { concentration in } \\
\text { the background } \\
\text { Ac,bg } \\
\text { MBq/ml } \\
\text { (measured with } \\
\text { an activity meter) }\end{array}$ & $\begin{array}{c}\text { Activity } \\
\text { concentration in the } \\
\text { background } \\
\text { ac,bg (MBq/ml) } \\
\text { (measured in the } \\
\text { image with } \\
\text { Q.Metrix, average of } \\
\text { the 5 ROIs) }\end{array}$ \\
$\begin{array}{l}\text { camera, } \\
\text { medical } \\
\text { centre }\end{array}$ & 1.19 & $4 \%$ & $-1.02 \%$ & 0.05 & 0.06 \\
\hline $\begin{array}{l}\text { Discovery } \\
\text { 670, } \\
\text { Department 1 }\end{array}$ & 1.13 & $10 \%$ & $-0.45 \%$ & 0.05 & 0.06 \\
\hline $\begin{array}{l}\text { NM/CT 850 } \\
\text { Department 2 }\end{array}$ & & & & & \\
\hline
\end{tabular}


Table 1. Calibration factor (Bg. cal), coefficient of variation (COV), total activity deviation ( $\triangle A$ tot), background activity concentration $(A c, b g)$ measured with the activity meter and background activity concentration (ac,bg, average of the 5 ROIs) measured in the image with Q.Metrix for two SPECT/CT acquisitions of a cylindrical homogeneous phantom in two nuclear medicine departments.

2. Accuracy of measurement for the activity of hot spherical inserts in the anthropomorphic phantom.

A) Figure 4 presents the accuracy of measurements for activity concentration in 6 hot spherical inserts of the NEMA IEC Body Phantom recorded with the Discovery NM/CT 670 gamma camera in Department 1 . The highest accuracy of measurement for activity concentration was obtained when using an image reconstruction technique with 24 iterations and 8 subsets, attenuation correction (AC), scatter correction (SC), and resolution recovery (RR). High levels of image noise after 24 iterations eliminated this reconstruction technique from clinical use. Another two reconstruction techniques offering the highest accuracy of measuring the activity concentration in spheres involved 5 iterations with 15 subsets and 4 iterations with 10 subsets. The use of reconstruction techniques with fewer than 4 iterations was associated with a significant reduction in the accuracy of measurement for the activity in all 6 spheres. The use of the reconstruction technique with just 2 iterations reduced the accuracy of measurement for the largest sphere $(\phi=37 \mathrm{~mm})$ by $8 \%$. When the Butterworth filter $(0.4810)$ was used in the technique with 2 iterations, the accuracy of activity measurement dropped by another $4 \%$ for this hot sphere. 


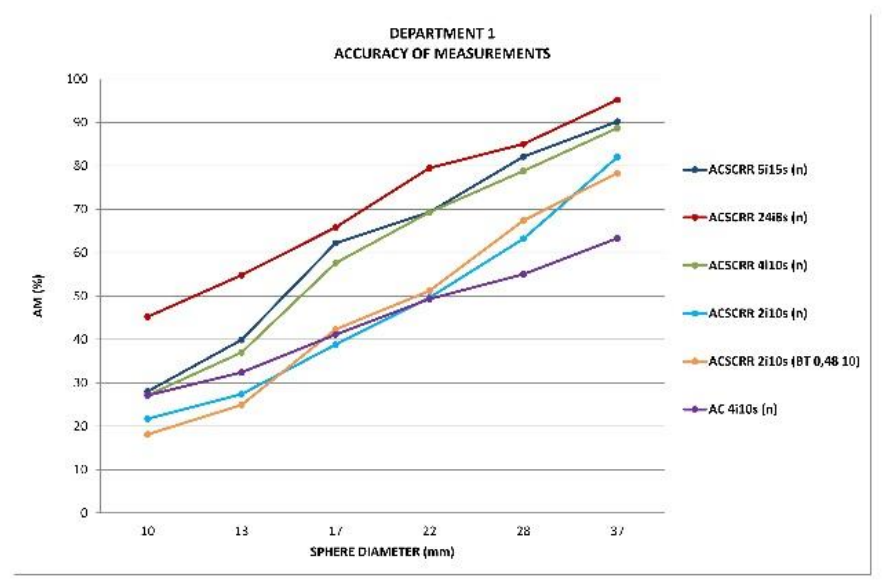

Fig. 4. Accuracy of measurements (AM) for activity in 6 spherical inserts of the NEMA IEC Body Phantom scanned with the Discovery NM/CT 670 gamma camera in Department 1.

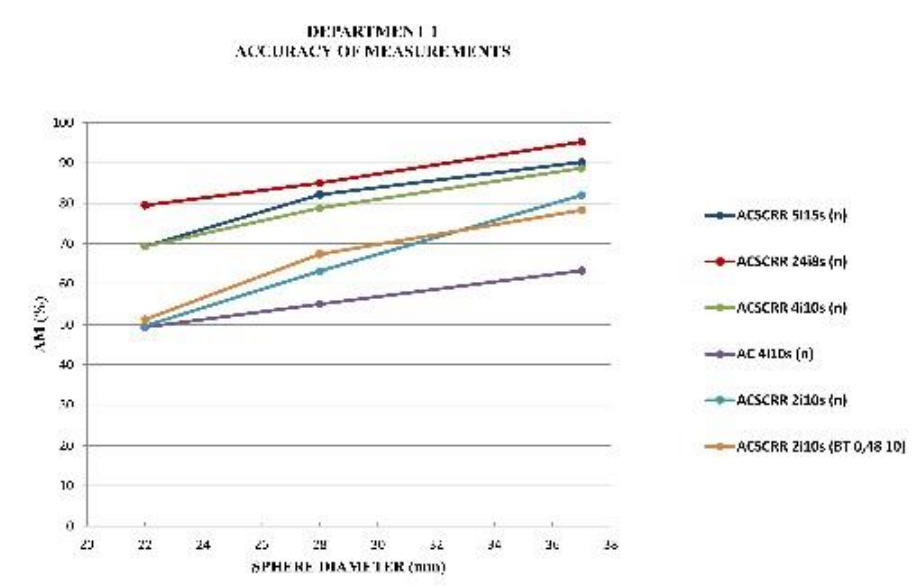

Fig. 5 A. Accuracy of measurements for activity in the 3 largest spherical inserts of the NEMA IEC Body Phantom scanned with the Discovery NM/CT 670 gamma camera in Department 1. 


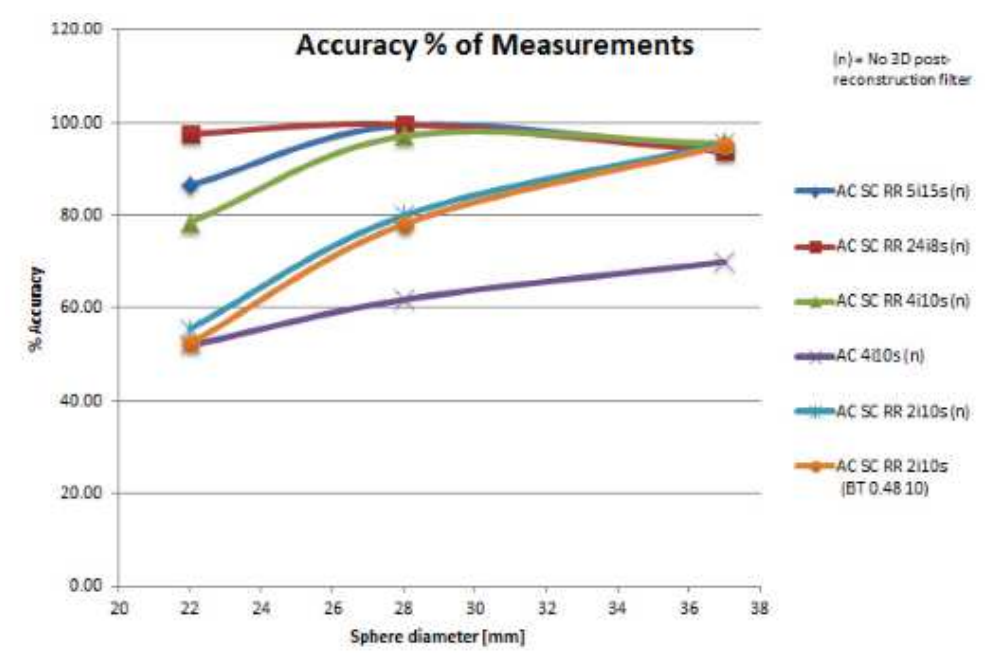

Fig. 5 B. Accuracy of measurements for activity in the 3 largest spheres of the NEMA IEC Body Phantom scanned in the GE Healthcare laboratory. Source: White Paper, DOC1951185, 2017, GE [4].

Figures 5 A, B compare the accuracy of measurements for activity concentration obtained for the 3 largest spheres of the anthropomorphic phantom scanned A) in clinical Department 1, B) in the GE Healthcare laboratory. For the two reconstruction techniques optimal for clinical use (5 i. 15 s. and 4 i. 10 s.) the accuracy of measurements for activity concentration in the largest sphere ( $\phi 37 \mathrm{~mm}$ ) was similar (approx. 90\%). For two smaller spheres ( $\phi 27$ and $22 \mathrm{~mm})$ the accuracy of measurement for activity concentration obtained in the clinical department of nuclear medicine was approx. 10\% lower than that reported by GE Healthcare.

Table 2 presents the calculated parameters of image quality obtained for the background and the $22 \mathrm{~mm}$ diameter spherical insert of the anthropomorphic phantom measured with two gamma cameras, Discovery NM/CT 670 and NM/CT 850. Activity concentration measured for the Tc99m solution in the $22 \mathrm{~mm}$ sphere was identical for both cameras. Hot contrasts measured in spherical inserts were comparable (60 vs 61\%). Activity concentrations measured in the background of the phantom in the two nuclear medicine departments were very similar. 


\begin{tabular}{|c|c|c|c|c|c|}
\hline PARAMETER & 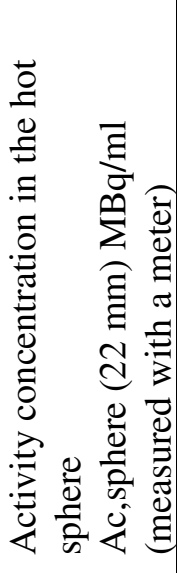 & 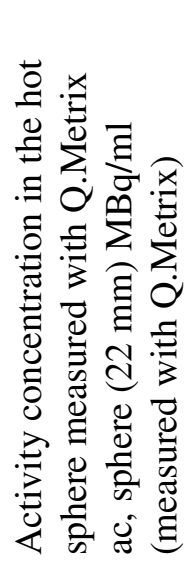 & 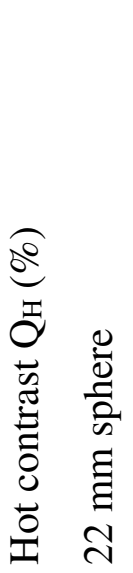 & 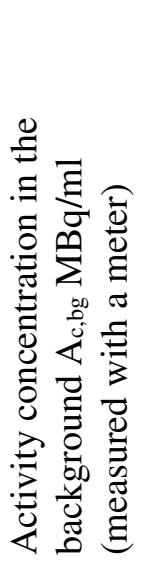 & 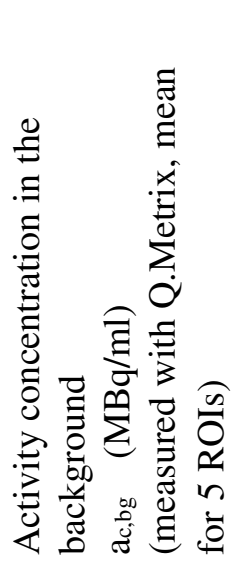 \\
\hline $\begin{array}{c}\text { Discovery } \\
\text { NM/CT } 670 \\
\text { Department } 1\end{array}$ & 0.32 & 0.20 & 60 & 0.04 & 0.06 \\
\hline $\begin{array}{c}\text { NM/CT } 850 \\
\text { Department } 2\end{array}$ & 0.32 & 0.20 & 61 & 0.04 & 0.05 \\
\hline
\end{tabular}

Table 2. Parameters of image quality obtained for the $22 \mathrm{~mm}$ diameter sphere and the background of the anthropomorphic phantom with two gamma cameras, Discovery NM/CT 670 and NM/CT 850.

B) Recovery coefficients RCmax.

Figure 6A presents Recovery coefficients (RCmax) for activity concentration in spheres of the anthropomorphic phantom. Images were acquired with the Discovery NM/CT 670 gamma camera from GE Healthcare and reconstructed with 7 techniques described in a paper by Gnesin et al. [2]. The type of a reconstruction technique had a minor effect on the accuracy of measurement for the smallest $(\phi 10 \mathrm{~mm})$ and largest $(\phi 37 \mathrm{~mm})$ spheres. $\mathrm{RC}_{\max }$ was in the range of 0.2-0.4 for the smallest sphere, and 1.3-1.4 for the largest sphere. The widest range of $\mathrm{RC}_{\max }$ (0.6- 1.6) was found for sphere no. 3 ( $\phi 17 \mathrm{~mm})$. 


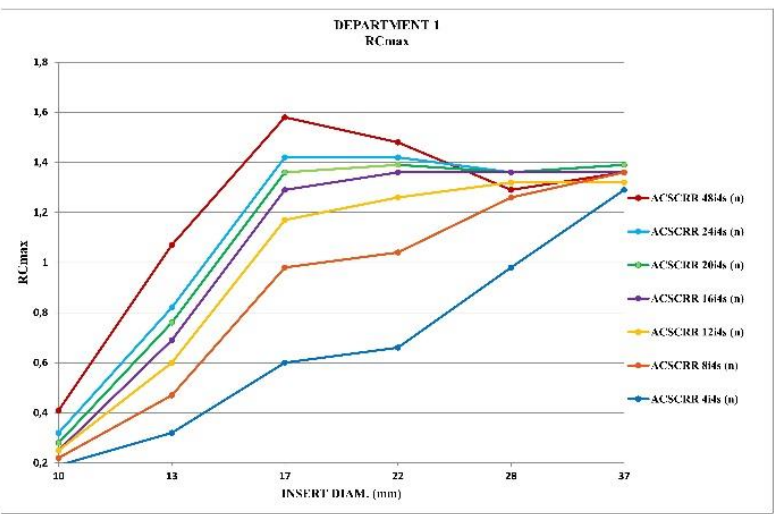

Fig. 6A. Recovery coefficient (RCmax) for activity concentration in 6 spherical inserts of the anthropomorphic phantom scanned with the Discovery NM/CT 670 gamma camera.

In the study by Gnesin et al. [2] the acquisitions of the anthropomorphic phantom were performed with a Siemens Symbia Intevo SPECT/CT system. Figure 6B presents recovery coefficients $\left(\mathrm{RC}_{\max }\right)$ for 6 spherical inserts reported by Gnesin et al. [2].

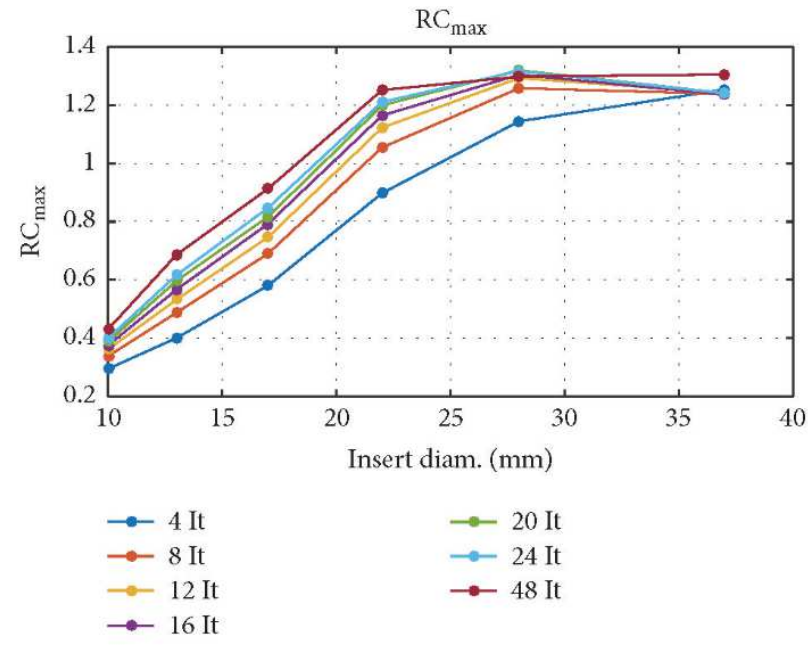

Fig. 6B. Recovery coefficients (RCmax) for activity concentration in 6 spherical inserts of the anthropomorphic phantom scanned with the Siemens Symbia Intevo SPECT/CT system [2]. (courtesy of Dr Silvano Gnesin).

3. Comparison of SUVs acquired for a single calibration source 
Table 3 presents the comparison of calculated SUVmax and SUVmean for a calibration source - a hot sphere $22 \mathrm{~mm}$ in diameter (an insert of the NEMA/IEC NU2 Body Phantom) registered with two different GE Healthcare gamma cameras in the two nuclear medicine departments. The activity of the calibration source at the time of acquisition start was $13.9 \mathrm{MBq}$ in Department 1 and 7.5 MBq in Department 2. Cross-section images of the calibration source were acquired using identical reconstruction parameters (4 iterations, 10 subsets, AC, SC, RR).

\begin{tabular}{|c|c|c|c|c|c|}
\hline GAMMA & $\begin{array}{c}\text { SPHERE } \\
\text { VOLUME } \\
\text { Q.Metrix } \\
(\mathrm{ml})\end{array}$ & $\begin{array}{c}\text { SPHERE } \\
\text { ACTIVITY } \\
\text { Q.Metrix } \\
(\mathrm{MBq})\end{array}$ & $\begin{array}{c}\text { SUV } \\
\text { mean } \\
(\mathrm{g} / \mathrm{ml})\end{array}$ & $\begin{array}{c}\text { SUV } \\
\mathrm{max} \\
(\mathrm{g} / \mathrm{ml})\end{array}$ & $\begin{array}{c}\text { SUV } \\
\mathrm{min} \\
(\mathrm{g} / \mathrm{ml})\end{array}$ \\
\hline $\begin{array}{c}\text { DISCOVERY } \\
670 \text { GE } \\
\text { Department } 1\end{array}$ & 5.8 & 8.17 & 52.40 & 113.30 & 7.18 \\
\hline $\begin{array}{c}\text { NM/CT } 850 \\
\text { Department 2 }\end{array}$ & 5.8 & 6.47 & 59.05 & 112.62 & 6.56 \\
\hline
\end{tabular}

Table 3. SUVmax and SUVmean for $\phi 22 \mathrm{~mm}$ sphere obtained using two gamma cameras: Discovery NM/CT 670 and NM/CT 850, in the two medical departments.

Conversion factors for SUVmax and SUVmean were calculated for the gamma camera systems used:

Conversion factor for SUVmax : $\frac{\text { NM/CT } 850 \text { DEPARTMENT } 2}{\text { DISCOVERY NM/CT } 670 \text { NM/CT } 850 \text { DEPARTMENT } 1}=0.99$

Conversion factor for SUVmean : $\frac{\text { NM/CT } 850 \text { DEPARTMENT } 2}{\text { DISCOVERY NM/CT } 670 \text { NM/CT } 850 \text { DEPARTMENT } 1}=1.13$

\section{Discussion}

1. Accuracy of activity recovery in a homogeneous phantom.

The calibration factors (Bg. cal) for the two gamma cameras, Discovery NM/CT 670 and NM/CT 850 , were not within $10 \%$ of the expected value (1), but were convergent, and only a $6 \%$ difference was found between them. Gnesin et al. [2] reported that the calibration factor (Bg. cal) was within $10 \%$ of the expected value, but the analysis was based on the images acquired during a two-times longer acquisition of the cylindrical homogeneous phantom.

The relatively low values of the coefficient of variation (COV\%) for the background proves the high degree of homogeneity of the solution filling the phantom, and thus the correct preparation of homogeneous phantoms in Department 1 and Department 2. Total activity deviation ( $\Delta$ Atot 
(\%)) for both gamma cameras was low, and activity concentrations measured in images with Q.Metrix were consistent.

Measurements taken in our experimental study confirmed the clinical suitability of 3 parameters of image quality (Bg. cal, COV and $\triangle \mathrm{Atot}$ ) for the validation of SPECT/CT system performance in terms of correct quantitative acquisitions of images.

2. Accuracy of measurement for the activity of hot spherical inserts in the anthropomorphic phantom.

A) The highest accuracy of measurement for the activity of hot spherical inserts was found in our study and by GE Healthcare [4] using an image reconstruction technique that involved 24 iterations, 8 subsets, and all types of corrections (attenuation correction, scatter correction, and resolution recovery). The algorithm with a large number of iterations significantly extended the reconstruction time, significantly increased the image noise, and was considered a technique unsuitable for clinical applications. The optimal reconstruction technique was the one with 5 iterations and 15 subsets. When 5 iterations were used, the maximum recovery coefficient (RCmax) for the activity measured in spherical inserts in the GE Healthcare laboratory was as high as $99.4 \%$ for the $28 \mathrm{~mm}$ sphere. In our study the accuracy of measurement for this sphere was $90.2 \%$. The lowest recovery coefficient (28\%) was found in our study for the smallest 10 mm diameter sphere. Laboratory tests described in the white paper (DOC1951185, 2017, GE Healthcare) did not evaluate the accuracy of recovery for the three smallest hot spherical inserts of the anthropomorphic phantom [4].

The use of reconstruction techniques with fewer than 4 iterations was associated with a significant reduction in the accuracy of activity measurement in all 6 spheres. The use of the reconstruction technique with just 2 iterations reduced the accuracy of measurement for the largest sphere $(\phi=37 \mathrm{~mm})$ by $8.2 \%$. When the Butterworth filter $(0.4810)$ was used in the technique with 2 iterations, the accuracy of activity measurement dropped by another $4 \%$ for this hot sphere.

Data presented in Table 2 imply that it is possible to obtain very repeatable results of the same tests and SPECT/CT acquisitions performed in two different medical departments with different gamma cameras. The anthropomorphic phantom was prepared and measured independently by different operators in the two different medical departments. Values of hot contrast $\left(\mathrm{QH}_{\mathrm{H}}\right)$ measured for the $\phi 22 \mathrm{~mm}$ hot spherical insert in these departments closely corresponded with values reported by Gnesin et al. [2]. 
B) The shapes of curves illustrating $\mathrm{RC}_{\max }$ obtained in our study are similar to those presented by Gnesin et al. [2]. RCmax curves were generated based on acquisitions from gamma cameras and acquisition software from different manufacturers. The analysis of curves revealed that the reconstruction technique with just 4 iterations and 4 subsets should not be used for the reconstruction of quantitative clinical acquisitions, regardless of the type of gamma camera system. Considering recovery coefficients $\mathrm{RC}_{\max }$ obtained in both studies, the optimal reconstruction technique is the one with 16 iterations and 4 subsets. The time of image reconstruction with this technique in Q.Metrix is not longer than 1.5 minutes.

\section{Comparison of SUVs acquired for a single calibration source}

Data presented in Table 3 were used to calculate conversion factors for SUV in order to compare standardized uptake values obtained in the two medical departments. The objective comparison of SUVs is very important for monitoring the status of a patient being tested in different nuclear medicine departments with different SPECT/CT scanners.

Currently, medical facilities worldwide do not perform the clinical standardization of SUVs as a clinical routine. For this reason, doctors are unable to analyse changes in the standardized uptake value for imaged tumours if the patient has been examined with different scanners, even in the same nuclear medicine department. Clinical publications reporting SUVmax and SUVmean measured in patients' images should provide SPECT/CT acquisition parameters, but also details regarding the image reconstruction technique $[5,6,7,8]$. This would allow for an initial comparison of quantification results for pathological changes in images acquired with different SPECT/CT systems.

\section{Conclusions}

1. Measurements taken in our study confirmed the clinical suitability of 5 parameters of image quality (Bg. cal- background calibration factor, $\Delta$ Atot- total activity deviation, COV- noise level estimation, Qн-hot contrast, AM-accuracy of measurements or RCrecovery coefficient) for the validation of SPECT/CT system performance in terms of correct quantitative acquisitions of images.

2. This work shows that absolute SPECT/CT quantification is achievable in clinical nuclear medicine centers. Results variation of quantitative analyzes between centers is mainly related to the use of different reconstruction methods. 
3. It is necessary to standardize the technique of measuring the SUV conversion factor obtained with different SPECT/CT scanners.

\section{List of abbreviations}

SPECT/CT: single photon emission computed tomography/computed tomography, GE: General Electric, NM: nuclear medicine, WCC: well counter calibration, AC: attenuation correction, SC: scatter correction, RR: resolution recovery, Bg. cal: background calibration factor, $\triangle$ Atot: total activity deviation, $\mathrm{COV}$ : noise level estimation, $\mathrm{Q}_{\mathrm{H}}$ : hot contrast, $\mathrm{AM}$ : accuracy of measurements, RC: recovery coefficient, SUV- standardized uptake value.

\section{Declarations}

1. Ethics approval and consent to participate.

Not applicable.

2. Consent for publication

Not applicable.

3. Availability of data and material

The datasets used and/or analyzed during the current study are available from the corresponding author on reasonable request.

4. Competing interests

The authors declare that they have no competing interests.

5. Founding

Not applicable.

6. Authors' contributions

HPB contributed to the conception, design of the study, data acquisition, paper drafting and revision. AS performed maesurements, took the lead in analysing the data. BB participated in paper drafting and revision. All authors read and approved the final manuscript.

7. Acknowledgement

Not applicable

\section{References}


1. Peters SMB, van der Werf NR, Segbers M, et al. Towards standardization of absolute SPECT/CT quantification: a multi-center and multi-vendor phantom study. EJNMMI Phys. 2019;6:29.

2. S. Gnesin, Ferreira PL, Malterre J, et al.. Phantom Validation of Tc-99m Absolute Quantification in a SPECT/CT Commercial Device, Hindawi Publishing Corporation Computational and Mathematical Methods in Medicine Volume 2016, Article ID 4360371, 6 pages.

3. National Electrical Manufacturers Association. NEMA Standards Publication NU 22007. Performance Measurements of Positron Emission Tomographs. Rosslin, VA, National Electrical Manufacturers Association, 2007.

4. NM Quantification. Q.Metrix for SPECT/CT Package. White Paper, DOC1951185, 2017, GE Healthcare.

5. Beck M, Sanders JC, Ritt P, et al. Longitudinal analysis of bone metabolism using SPECT/CT and 99mTc-diphosphono-propanedicarboxylic acid: comparison of visual and quantitative analysis. EJNMMI Res 2016;6(1):60.

6. Dong F, Li L, Bian Y, et al. Standardized Uptake Value Using Thyroid Quantitative SPECT/CT for the Diagnosis and Evaluation of Graves' Disease: A Prospective Multicenter Study. Biomed Res Int. 2019;2019:7589853. doi:10.1155/2019/7589853

7. Brady SL, Shulkin BL. Analysis of quantitative [I-123] mIBG SPECT/CT in a phantom and in patients with neuroblastoma. EJNMMI Phys 2019;6:31

8. de Laroche R, Bourhis D, Robin P, et al. Feasibility Study and Preliminary Results of Prognostic Value of Bone SPECT-CT Quantitative Indices for the Response Assessment of Bone Metastatic Prostate Carcinoma to Abiraterone. Frontiers in Medicine 2020;6:342. 


\section{Figures}

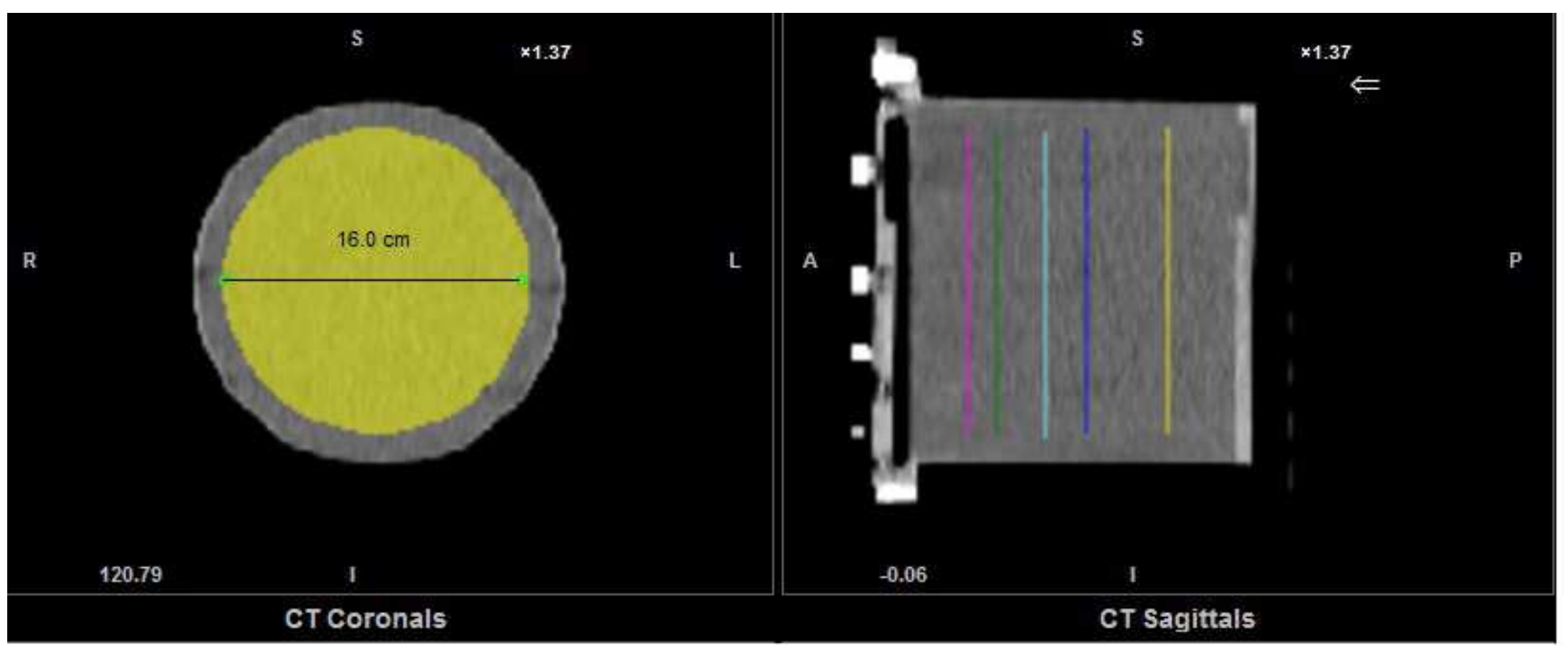

\section{Figure 1}

Image of the WCC phantom with 5 circular regions of interest of $16 \mathrm{~cm}$ in diameter located at different levels along the long axis of the phantom, reconstructed with Xeleris 2 from GE Healthcare.

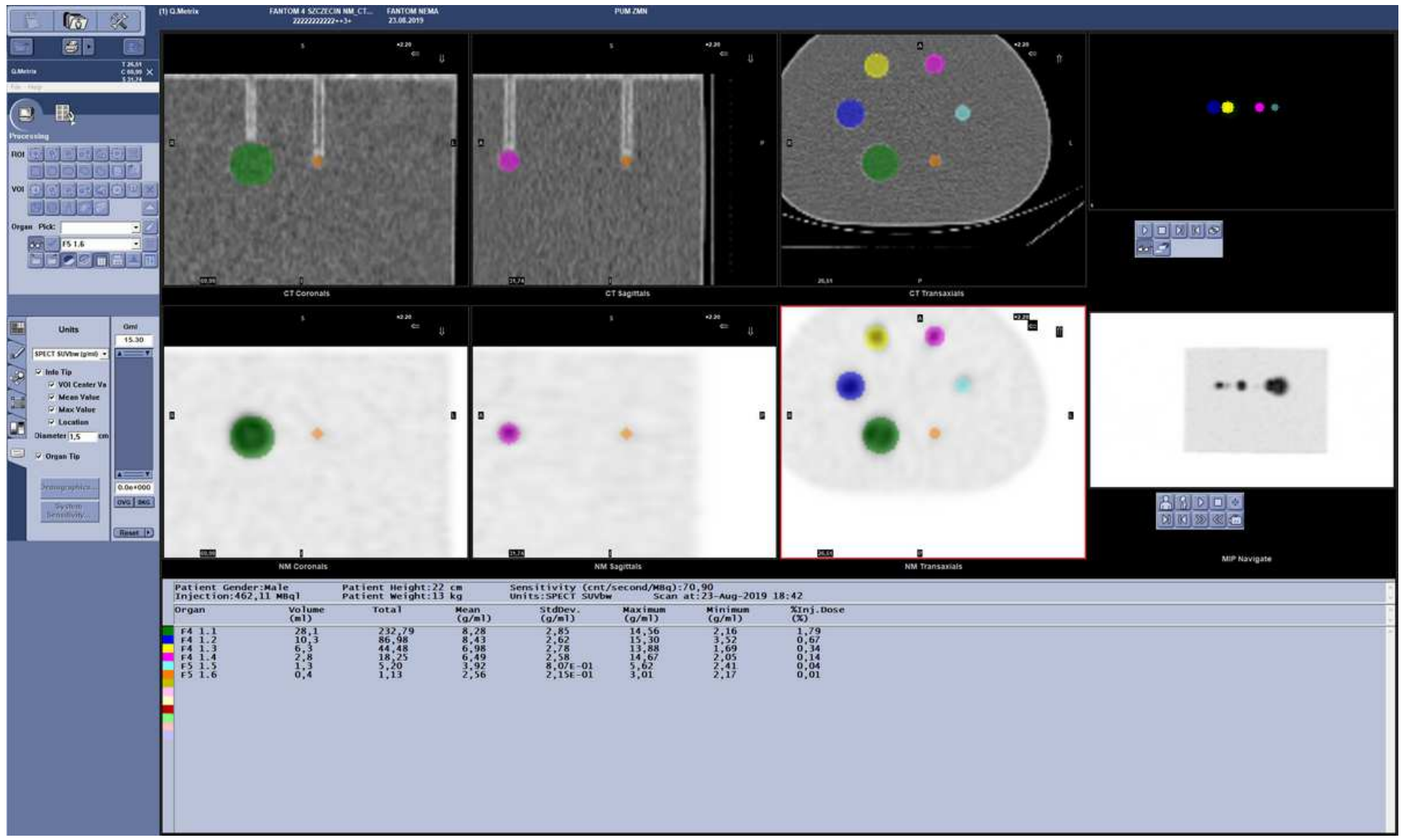

Figure 2 
A screenshot of the Q.Metrix software during the measurement of hot spheres activity in the crosssections of the anthropomorphic NEMA IEC Body Phantom. Colour rings correspond with VOI (Volume of Interest) for 6 hot spheres.

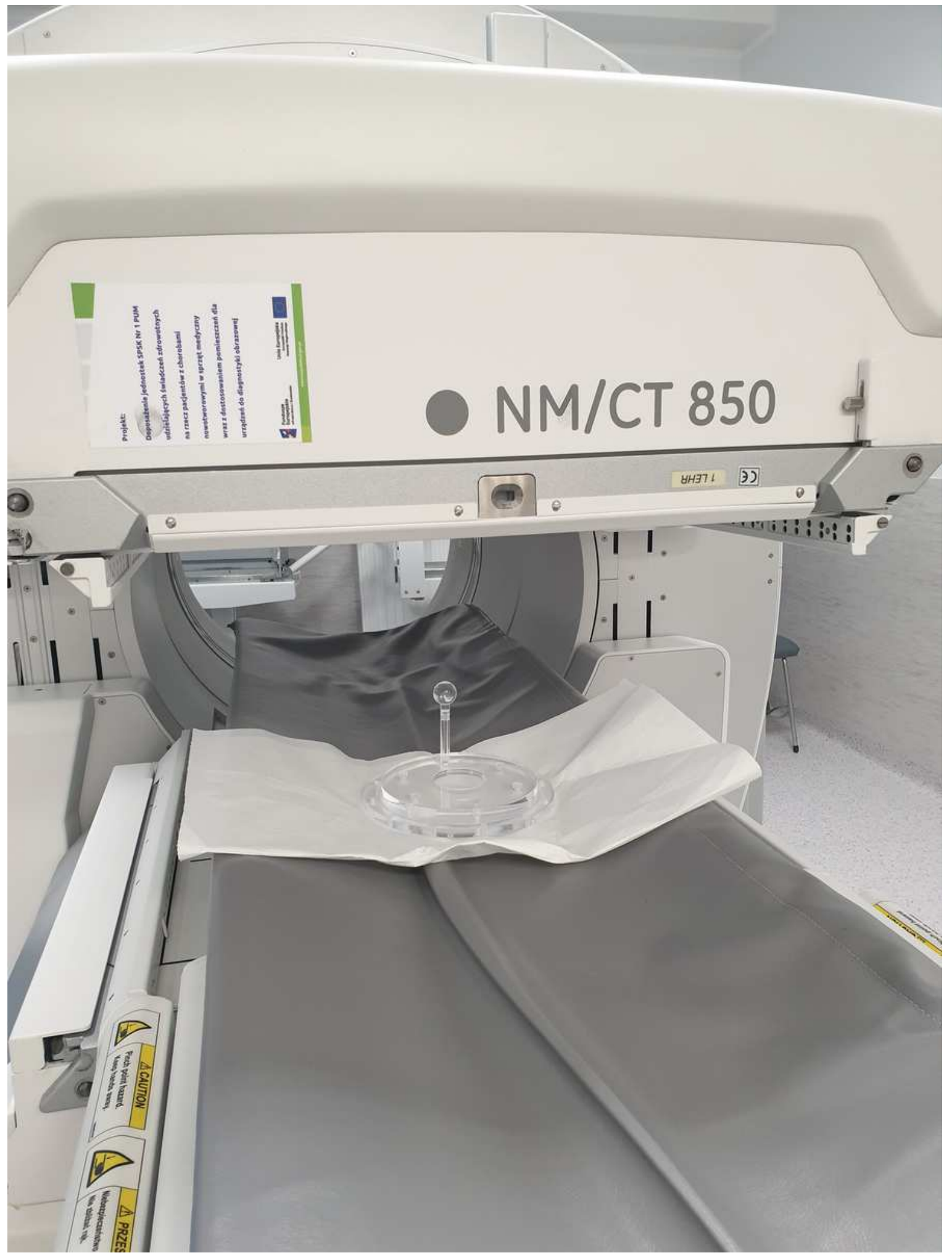

Figure 3

Acquisition of the calibration source in Department 2. 


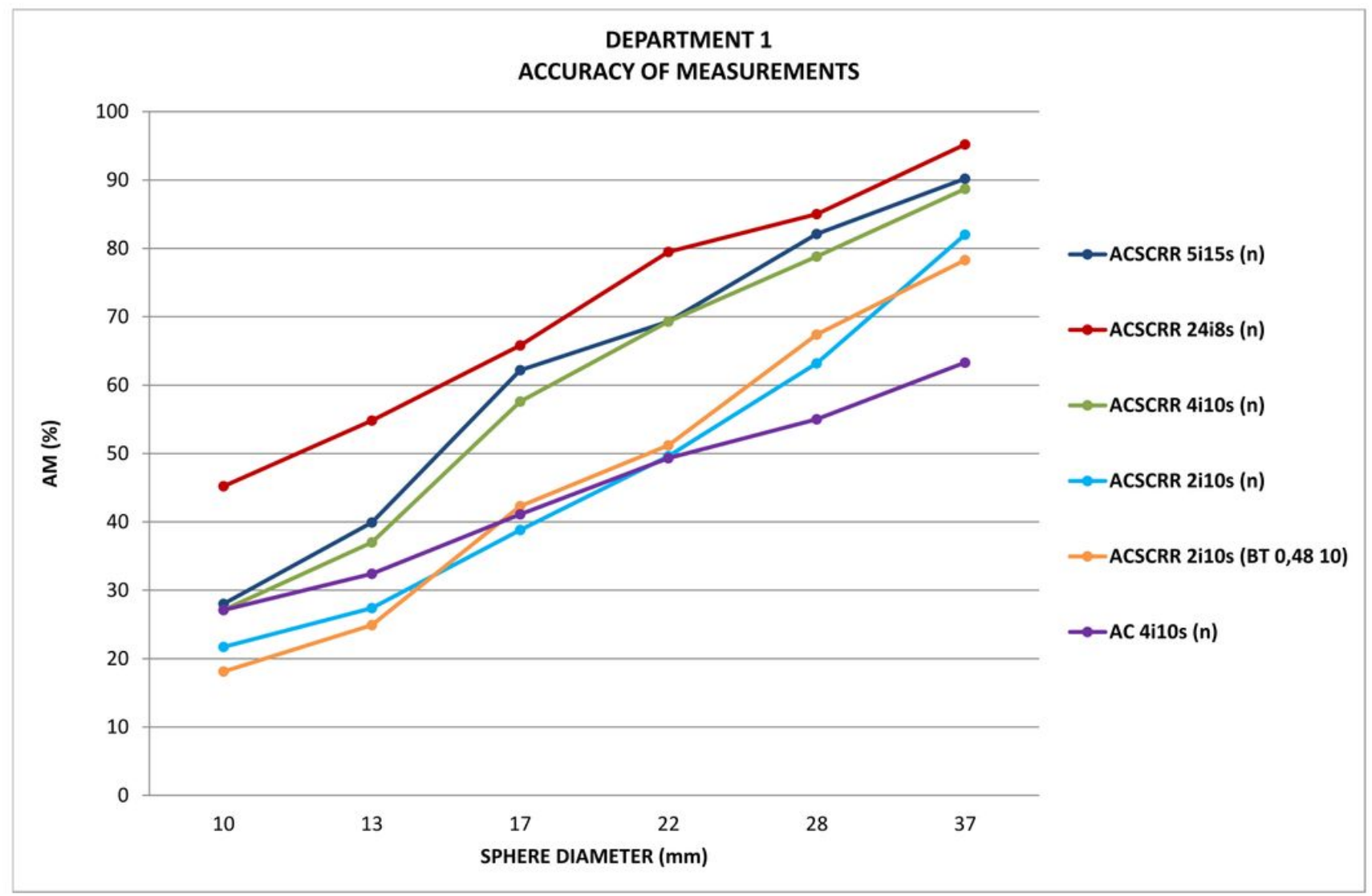

\section{Figure 4}

Accuracy of measurements (AM) for activity in 6 spherical inserts of the NEMA IEC Body Phantom scanned with the Discovery NM/CT 670 gamma camera in Department 1.

DEPARTMENT 1
ACCURACY OF MEASUREMENTS
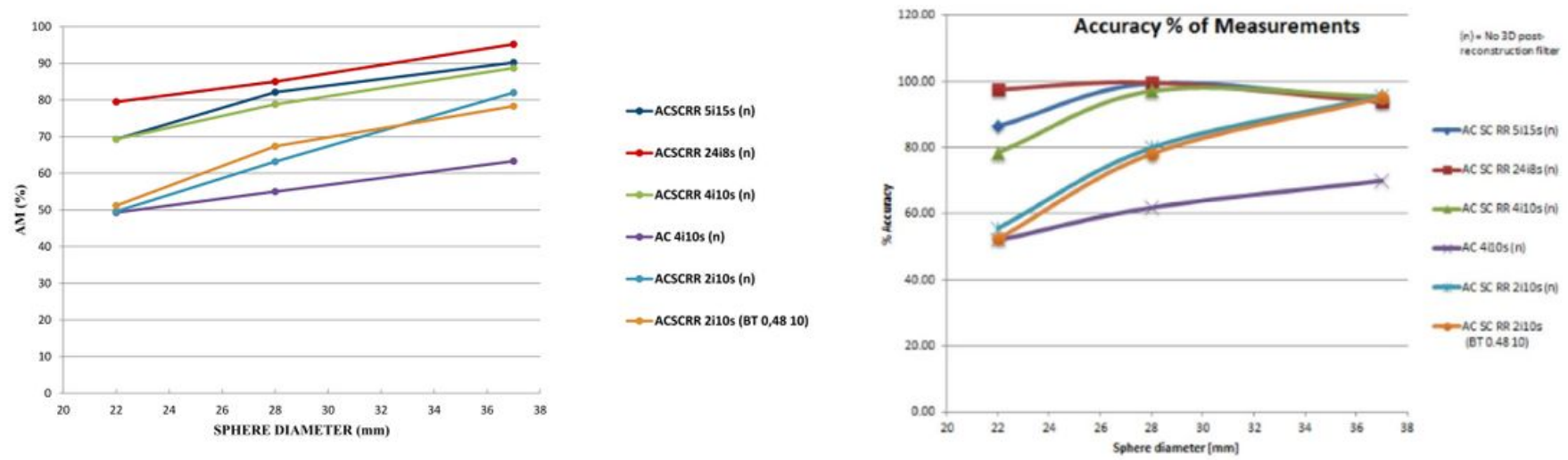


\section{Figure 5}

A. Accuracy of measurements for activity in the 3 largest spherical inserts of the NEMA IEC Body Phantom scanned with the Discovery NM/CT 670 gamma camera in Department 1. B. Accuracy of measurements for activity in the 3 largest spheres of the NEMA IEC Body Phantom scanned in the GE Healthcare laboratory. Source: White Paper, DOC1951185, 2017, GE [4].
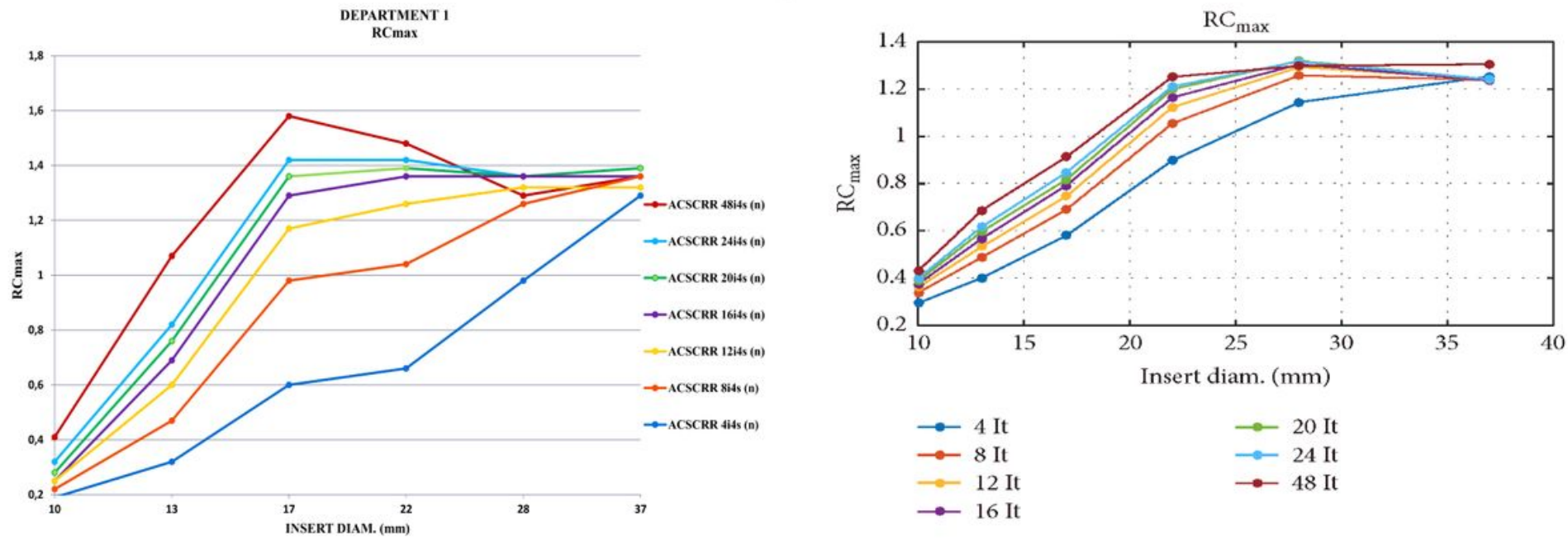

\section{Figure 6}

A. Recovery coefficient (RCmax) for activity concentration in 6 spherical inserts of the anthropomorphic phantom scanned with the Discovery NM/CT 670 gamma camera. B. Recovery coefficients (RCmax) for activity concentration in 6 spherical inserts of the anthropomorphic phantom scanned with the Siemens Symbia Intevo SPECT/CT system [2]. (courtesy of Dr Silvano Gnesin). 